\title{
4 Capacidade funcional de idosos acompanhados pela Fisioterapia de uma Unidade de Atenção Primária à Saúde
}

\author{
Functional capacity of the elderly \\ accompanied by physiotherapy in \\ a Primary Health Care Unit
}

FisiSenectus. Unochapecó Ano 7, n. 2 - Jul/Dez. 2019 p. $23-38$

\section{Carine Sousa dos Santos. carine.sousas@gmail.com}

Fisioterapia pelo Centro Universitário UniFanor Wyden (2016.1). Especialista em Saúde da Família pela Universidade da Integração Internacional da Lusofonia Afro-Brasileira (2018). Residente em Saúde da Família e Comunidade - Residência Integrada em Saúde da Escola de Saúde Pública do Ceará (em conclusão).

\section{Patrícia Moreira Costa Collares.pmcollares@yahoo.com.br}

Fisioterapeuta pela Universidade de Fortaleza (bolsista CNPq - 2007), Especialista em Fisioterapia Respiratória e Cardiovascular (2009), Especialista em Gestão e Docência do Ensino Superior (2011), Mestre em Saúde Coletiva (bolsista Capes). Doutora em Saúde Coletiva (Unifor/UFC/UECE), bolsista Funcap, durante o ano de 2011. Professora Adjunto (DE) da Universidade Federal do Ceará (UFC) e no Mestrado em Saúde da Família (RENASF/Fiocruz/UFC).

Anairtes Martins de Melo.meloanairtes@yahoo.com.br

Fisioterapeuta pela Universidade de Fortaleza (1998), Mestre em Ensino na Saúde pela Universidade Estadual do Ceará (2015). Atualmente, é professora do Centro Universitário UniFanor Wyden, no curso de graduação em Fisioterapia.

\section{Gerídice Lorna Andrade de Moraes. geridice@hotmail.com}

Enfermeira pela Universidade Estadual do Ceará (1997), graduada em Licenciatura Plena em Enfermagem pela Universidade Estadual do Ceará (1998), Mestre em Enfermagem pela Universidade Federal do Ceará (UFC), Doutora em Enfermagem pela UFC, Especialista em Gerontologia Titulada pela Sociedade Brasileira de Geriatria e Gerontologia (SBGG), Especialista em Saúde da Família pela Escola de Saúde Pública, Articuladora da Atenção Primária à Saúde do município de Fortaleza (SMS). Docente do Centro Universitário Christus e da Universidade de Fortaleza. Pesquisadora responsável pelo Grupo de Estudo e Pesquisa em Gestão de Enfermagem (GEPGENF). Professora do curso de pós-graduação em Gerontologia da UniAteneu, professora do curso de Especialização em Saúde do Idoso da Universidade Estadual do Ceará. Conselheira Titular do Conselho Nacional de Saúde (CNS), Titular do Conselho Municipal do Idoso.

Maria Raquel Lima Lacerda. raquellacerda2810@gmail.com

Nutricionista pela Universidade Estadual do Ceará. Residência em Saúde da Família e Comunidade pela Escola de Saúde Pública do Ceará (ESP-CE) pela Residência Integrada em Saúde (RIS). Diretora de Administração do Sindicato dos Nutricionistas do Estado do Ceará (Sindnuce).

Danielle Hortencio Pereira dos Santos.daniellehp@unifor.br

Fisioterapeuta pela Universidade de Fortaleza (Unifor). Fisioterapeuta do Núcleo Ampliado de Saúde da Família e Atenção Básica do Município de Fortaleza/CE e do Núcleo de Atenção Médica Integrada (NAMIUnifor) e preceptora do Núcleo de Fisioterapia pela Residência Integrada em Saúde da Escola de Saúde Pública do Ceará (RIS/ESP-CE), na ênfase Saúde da Família e Comunidade. 


\begin{abstract}
Resumo
Introdução: a avaliação da capacidade funcional obtém foco no exame do avaliado e objetiva indicar o nível máximo de funcionalidade que um indivíduo pode atingir em um dado domínio em dado momento, é um indicador de saúde que se relaciona com a qualidade de saúde e aspectos biopsicossociais. Objetivo: avaliar a capacidade funcional dos idosos acompanhados por uma unidade de Atenção Primária à Saúde pela Fisioterapia e descrever o perfil sociodemográfico e familiar e os dados antropométricos dos entrevistados. Materias e métodos: realizou-se um estudo transversal, descritivo, com abordagem quantitativa no período de dezembro de 2018 a fevereiro de 2019 em Fortaleza/CE. Coletaram-se dados de 35 idosos utilizando um questionário acerca do perfil sociodemográfico e familiar dos pesquisados e a Short Physical Performance Battery. Resultados: na amostra, houve predomínio de mulheres, entre 60 e 69 anos, casadas, domésticas e sedentárias. A maioria das entrevistadas está com sobrepeso, entretanto a minoria apresentou sarcopenia. Quanto à capacidade funcional, predomina moderado desempenho físico, tendo o pior resultado a etapa de sentar-levantar do teste. Conclusões: a avaliação da capacidade funcional demonstrou a extratificação de idosos em vulnerabilidade física, requerendo o desenvolvimento de ações de intervenção em grupos ou projetos terapêuticos no território
\end{abstract}

\title{
Palavras-chave
}

Idoso; Atenção Primária à Saúde; Fisioterapia.

\begin{abstract}
Introduction: the assessment of functional capacity focuses on the examination of the subject and aims to indicate the maximum level of functionality that an individual can achieve in a given domain at a given time, is a health indicator that relates to quality of health and biopsychosocial aspects. Objective: to evaluate the functional capacity of the elderly followed by a Primary Health Care Unit through Physical Therapy and describe the sociodemographic, family and anthropometric data of the interviewees. Materials and methods: a cross-sectional, descriptive study with a quantitative approach was performed from December 2018 to February 2019 in Fortaleza / Ce. Data were collected from 35 elderly people using a questionnaire about the sociodemographic and family profile of the respondents and the Short Physical Performance Battery. Results: in the sample there was a predominance of women, between 60 and 69 years old, married, domestic and sedentary. Most of the interviewees are overweight, however the minority had sarcopenia. As for functional capacity, moderate physical performance predominates, with the worst result being the sitting of the test. Conclusions: the evaluation of functional capacity demonstrated the extratification of elderly in physical vulnerability, requiring the development of intervention actions in groups or therapeutic projects in the territory.
\end{abstract}

\section{Keywords}

Elderly; Primary Health Care; Physiotherapy.

\section{Introdução}

$\infty \infty \infty \times \infty \times \infty \times \infty \times \infty \times \infty \times \infty \times \infty \times \infty \times \infty \times \infty)$

Desde 1940, o Brasil passa por transformações no padrão demográfico, no qual observam-se, na população idosa, as taxas mais altas de crescimento populacional. De acordo com o Instituto Brasileiro de Geografia e Estatística, os idosos representavam $10 \%$ da totalidade populacional em 2010; já para 2030, a expectativa é de que esse número passe para $19 \%{ }^{1}$.

Observando os últimos três censos brasileiros, o estado do Ceará reflete o ritmo de envelhecimento da população brasileira, pois a proporção de idosos aumentou, passando de 7,8\% (1991) para 8,9\% (2000) e 10,8\% (2010). 0 índice de enveIhecimento - número de pessoas com 60 anos ou mais de idade, para cada 100 pessoas menores de 15 anos de idade - evolui significativamente, nos 
censos de 1991,2000 e 2010 , de $19,9 \%$ para $26,4 \%$ e $41,6 \%$, na mesma ordem².

Percebe-se que o perfil do idoso, no Brasil, possui algumas particularidades, entre elas: maior proporção de mulheres; residência em domicílios multigeracionais, cuja renda familiar corresponde à renda individual (aposentadoria) do idoso; maior exposição a doenças crônicas não transmissíveis, o que traz como consequência a presença de sequelas limitantes do desempenho funcional, acarretando uma população de pessoas idosas frágeis com demanda de cuidados especiais, principalmente no que tange à realização de atividades de vida diária ${ }^{3}$.

Muitos idosos têm o Sistema Único de Saúde (SUS) como principal meio de acesso ao serviço de saúde. A Atenção Primária em Saúde (APS) é a principal porta de entrada no SUS, sendo centro de comunicação da Rede de Atenção à Saúde, coordenadora e ordenadora do cuidado, das ações e dos serviços disponíveis na rede. Na APS, o Núcleo Ampliado de Saúde da Família e Atenção Básica (NASF-AB) é composto por uma equipe multiprofissional e interdisciplinar com diferentes categorias profissionais que atua de maneira integrada para dar suporte aos profissionais da equipe de Saúde da Família (eSF) ${ }^{4}$.

Quando inserido no NASF-AB, o fisioterapeuta participa com seu conhecimento do núcleo profissional com o intuito de contribuir com as demandas que emanam do território, que podem ser casos de perda de funcionalidade e/ou situações que produzem barreiras para a independência física dos sujeitos. Diante das mudanças do perfil demográfico da população brasileira, é imprescindivel que o profissional fisioterapeuta utilize ferramentas para identificação de usuários idosos com redução de mobilidade, alterações cognitivas e limitações funcionais ${ }^{5}$.

A avaliação da capacidade funcional obtém foco no exame do avaliado e objetiva indicar o nível máximo de funcionalidade que um indivíduo pode atingir em um domínio em dado momento. É um indicador de saúde que se relaciona com a qualidade de saúde e aspectos biopsicossociais e tornou-se indispensável para a escolha da intervenção que busque minimizar os efeitos da incapacidade física ou preveni-la no âmbito da APS ${ }^{1,6}$.
Diante do exposto, justifica-se a pesquisa devido ao envolvimento da autora com a temática durante a vivência como profissional residente em uma Unidade de Atenção Primária à Saúde (UAPS) em Fortaleza/CE, onde foi possível observar que a população idosa apresenta um elevado quantitativo de demandas para a Fisioterapia do NASF-AB. Além disso, salienta-se a importância do estudo para a ampliação do conhecimento sobre a capacidade funcional de idosos.

Ressalta-se a contribuição da pesquisa para a comunidade científica ao disseminar a avaliação de idosos embasada na funcionalidade humana e no perfil do público para a assistência da Fisioterapia no serviço público de saúde.

Objetivou-se avaliar a capacidade funcional dos idosos acompanhados pela Fisioterapia do NASF-AB de uma UAPS de Fortaleza, bem como descrever o perfil sociodemográfico e familiar e os dados antropométricos da população estudada.

\section{Materiais e Métodos}

$\infty \times \infty \times \infty \times \infty \times \infty \times \infty \times \infty \times \infty \times \infty \times \infty \times \infty \times \infty \times \infty \times$

Trata-se de um estudo de abordagem quantitativa, do tipo descritivo e transversal. A coleta de dados ocorreu entre dezembro de 2018 e fevereiro de 2019 e foi realizada na UAPS Aída Santos e Silva, localizada na Secretaria Executiva Regional (SER) II de Fortaleza/CE.

A SER II de Fortaleza é formada por 20 bairros, onde habitam 334.868 pessoas, correspondendo a $13,50 \%$ da população da capital. A Unidade de Saúde Aída Santos e Silva está localizada no bairro Vicente Pinzón e, segundo o sistema de informação do município, Fastmedic ${ }^{\circledR}$, conta com três equipes da eSF e tem uma população total de responsabilidade sanitária de 16.425 usuários.

Foram incluídos na pesquisa os sujeitos com idade igual ou superior a 60 anos com capacidade de compreensão e deambulação, podendo utilizar auxílio de marcha, exceto cadeiras de rodas, que foram encaminhados para a Fisioterapia, no período de abril a outubro de 2018. 0 critério de exclusão foi mudança de endereço do usuário para área não adscrita da UAPS, depois da realização 
do encaminhamento, deixando de ser vinculado à unidade de saúde em questão.

Na unidade de saúde em estudo, a necessidade de acompanhamento da Fisioterapia obedeceu ao Fluxo de Encaminhamento para o NASF-AB. Tal processo de trabalho foi acordado com antecedência entre a equipe de profissionais residentes, a gestão da unidade de saúde e demais profissionais do serviço; para tanto, as demandas eram escritas em fichas padrões, em que eram descritas: a identificação do usuário, a justificativa para o encaminhamento com referência à fisioterapeuta e a identificação de qual categoria profissional referenciou o paciente. Os encaminhamentos poderiam partir dos profissionais da eSF (enfermeiras, médicos e agentes comunitários de saúde) e de outras categorias profissionais do NASF da unidade (psicólogo, nutricionista e assistente social).

Com o intuito de esclarecer os objetivos da pesquisa e os riscos, os usuários foram encaminhados para consulta com a fisioterapeuta residente. Os agendamentos foram entregues aos usuários pelos seus respectivos ACS, como ocorre rotineiramente na unidade. Quando de acordo a participar, assinou-se o Termo de Consentimento Livre e Esclarecido, seguindo a Resolução n 466/12, do Conselho Nacional de Saúde.

A coleta de dados foi realizada em uma única avaliação individual com duração de 50 minutos. A primeira parte do instrumento aplicado para coleta de dados baseou-se na $4^{\mathrm{a}}$ edição da $\mathrm{Ca}$ derneta de saúde da pessoa idosa do Ministério da Saúde ${ }^{7}$. Realizou-se a coleta de dados pessoais (identificação, estado civil, profissão/ocupação e escolaridade) e informações sociofamiliares (renda financeira e outras informações sobre apoio familiar/social).

Foi realizada a mensuração do peso e altura em uma balança antropométrica mecânica 150 kg MIC-33-Micheletti ${ }^{\circledR}$, com o indivíduo permanecendo estático, em pé, sem uso de acessórios, com os braços estendidos ao longo do corpo e a cabeça erguida. O IMC foi calculado pela relação entre a massa corporal e a estatura do indivíduo ${ }^{8}$. 0 escore do IMC foi classificado de acordo com a Caderneta de saúde da pessoa idosa do Ministério da Saúde, sendo considerado baixo peso valores menores que $22 \mathrm{~kg} / \mathrm{m}^{2}$; de $22 \mathrm{~kg} / \mathrm{m}^{2}$ a $27 \mathrm{~kg} / \mathrm{m}^{2}$, o peso foi considerado adequado; e escore maior que $27 \mathrm{~kg} / \mathrm{m}^{2}$ indicou sobrepeso ${ }^{7}$.

Também em conformidade com a Caderneta de saúde da pessoa idosa, utilizou-se a medida do perímetro da panturrilha esquerda para avaliação da massa muscular nos idosos. Para tanto, os entrevistados estiveram sentados com os joelhos fletidos a $90^{\circ}$ e os pés apoiados no solo, e a avaliadora utilizou uma fita métrica inelástica para fazer a medição na parte mais protuberante da panturriIha. Medidas menores que $31 \mathrm{~cm}$ foram consideradas indicativo de redução de massa muscular, estando associadas a maior risco de quedas e dependência funcional ${ }^{7}$.

Para avaliação da capacidade funcional, aplicou-se a Short Physical Performance Battery (SPPB), um instrumento prático e eficaz para a avaliação da funcionalidade, como também para o rastreio de riscos futuros de incapacidades ${ }^{9}$. Essa bateria de testes foi escolhida devido à sua aplicabilidade no ambiente do posto de saúde, exigindo instrumentos simples (cadeira, fita métrica e cronômetro) e valendo-se do espaço físico disponível na unidade.

A SPPB consiste na avaliação de três itens: equilíbrio estático em pé, velocidade de marcha em passo habitual e habilidade de levantar-se de uma cadeira. Para tanto, o material necessário é uma cadeira e um cronômetro em um espaço de quatro metros. Cada item avaliado varia de 0 a 4 pontos, somando o escore total de 0 a 12 pontos. 0 somatório é interpretado da seguinte maneira: 0 a 3 pontos indica incapacidade ou capacidade ruim; 4 a 6 pontos, baixa capacidade; 7 a 9 pontos, capacidade moderada; e 10 a 12 pontos, boa capacidade ${ }^{9}$.

Os dados foram processados utilizando o Microsoft Office Excel 2010 para posterior análise a partir da construção de gráficos e tabelas, sendo estes apresentados em números absolutos e porcentagem. A demonstração dos dados atendeu aos preceitos da estatística descritiva, utilizando a categorização dos resultados provenientes da avaliação física, bem como da aplicação do SPPB e dos demais itens da coleta de dados, para, posteriormente, tais dados foram serem confirmados ou refutados pela literatura atualizada e pertinente à temática. 
0 projeto de pesquisa foi apreciado e aprovado pelo Comitê de Ética em Pesquisa da ESP/CE, sob o Parecer Consubstanciado n 3.059.656 e Certificado de Apresentação para Apreciação Ética (CAAE) 03585718.0 .0000 .5037$.

\section{Resultados}

$\infty \times \infty \times \infty \times \infty \times \infty \times \infty \times \infty \times \infty \times \infty \times \infty \times \infty \times \infty)$

Cinquenta e oito usuários idosos realizaram agendamento para Fisioterapia, sendo que o percentual de encaminhamentos por profissionais foi: 48,2\% $(n=28)$ de médicos, 32,7\% $(n=19)$ de Agentes Comunitários de Saúde, 15,5\% ( $n=9)$ de enfermeiras e 3,4\% (n=2) da nutricionista e assistente social do NASF. Destes, foram avaliados 35 usuários, pois 23 não compareceram na unidade na data do agendamento.

Dos 35 idosos avaliados, 94,3\% ( $n=33)$ eram do sexo feminino, com predomínio da faixa etária de 60 a 69 anos, 68,5\% ( $n=24)$. Quanto à cor/raça, $34,2 \%(n=12)$ consideram-se pardos(as) e declararam-se casados(as) 34,2\% ( $n=12)$, dados representados na Tabela 1.

Dando continuidade à descrição da Tabela 1 , sobre o nível de escolaridade dos entrevistados, $37,1 \%(n=13)$ frequentaram de quatro a sete anos a escola convencional. Entre as idosas, a maioria era/é doméstica/secretária do lar, 31,4\% $(n=11)$, e os idosos eram/são pescadores e motoristas, $2,8 \%(n=1)$. Sabe-se que $85,7 \%(n=30)$ não trabalham atualmente, mas somente $57,1 \%$ dos(as) idosos(as) ( $n=20)$ são aposentados(as) ou pensionistas. A maioria, $77,1 \%(n=27)$, não recebe Benefício de Prestação Continuada (BPC), e 82,8\% (n=29) não recebe Bolsa Família.

Quanto ao arranjo familiar, $11,4 \%(n=4)$ dos idosos moram sozinhos, $74,2 \%(n=26)$ afirmaram que moram com familiares como filhos, primos, irmãos, entre outros, e 37,1\% ( $n=13)$ certificaram que moram com cônjuge ou companheiro(a). Quando perguntados se nos últimos 30 dias teriam se encontrado com amigos ou familiares para conversar ou fazer alguma atividade, como ir ao cinema ou à igreja, passear ou caminhar juntos, $80 \%(n=28)$ responderam que sim e $94,2 \%$ $(n=33)$ afirmaram que contam com alguém para acompanhá-los(as) à unidade de saúde ou consulta, em caso de necessidade (Tabela 2 ).
Dando continuidade à descrição da Tabela 2, $82,8 \%(n=29)$ dos entrevistados declararam que têm fácil acesso aos estabelecimentos comerciais (farmácias/padaria/supermercado) e 77,1\% ( $n=27$ ) afirmaram que têm fácil acesso ao transporte público (ônibus).

Acerca dos hábitos de vida, somente $17,1 \%$ $(n=6)$ dos(as) idosos(as) praticam atividade física pelo menos três vezes por semana. Atualmente, $97,1 \%(n=34)$ não fumam produto à base de tabaco; desses, $52,9 \%(n=18)$ já fizeram uso abusivo de tabaco. Sobre o uso de bebida alcóolica, 97,1\% $(n=34)$ não consomem e somente $8,5 \%(n=3)$ já fizeram uso abusivo de álcool (Tabela 3 ).

Quanto ao IMC, apenas $31,4 \%(n=11)$ dos entrevistados apresentaram IMC adequado, 62,8\% $(n=22)$ foram classificados com sobrepeso e, em baixo peso, estavam $5,7 \%(n=2)$. Percebe-se, entre os idosos, dificuldade em conhecer o peso corporal habitual, pois $28,5 \%(n=10)$ não souberam informar ou não lembraram tal quesito. Dos idosos que responderam, 32\% $(n=8)$ apresentaram peso corporal atual, confirmado por balança, maior que o peso dito como habitual. Entre os idosos que mostraram perda de peso, identificou-se somente uma $(2,8 \%)$ idosa com perda de peso não intencional maior de $4,5 \mathrm{~kg}$. Neste trabalho, somente $14,2 \%$ $(n=5)$ das idosas tiveram medidas da circunferência da panturrilha esquerda abaixo de $31 \mathrm{~cm}$, evidenciando sarcopenia. Tais dados são expressos na Tabela 4.

$\mathrm{Na}$ Tabela 5, apresentam-se os resultados da pontuação do teste de capacidade funcional. Do total, $20,1 \%(n=7)$ demonstraram bom desempenho (de 10 a 12 pontos), 51,4\% ( $n=18)$ dos avaliados apresentaram moderado desempenho (7 a 9 pontos), seguidos de $22,8 \%(n=8)$ com baixo desempenho (4 a 6 pontos), e somente $5,7 \%(n=2)$ dos idosos apresentaram incapacidade ou desempenho muito ruim ( 0 a 3 pontos).

\section{Discussão}

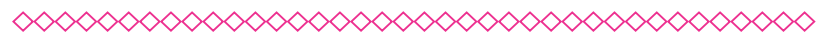

A capacidade funcional guarda relação com uma série de fatores sociodemográficos, familiares e ocupacionais e características físicas 
expressas em medidas antropométricas. Segundo a Organização Mundial de Saúde, a funcionalidade humana é influenciada por aspectos estruturais e funcionais dos sistemas biológicos, como também por elementos que englobam a totalidade das experiências vivenciadas pelo sujeito ${ }^{6}$.

Entre os dados sociodemográficos da pesquisa, constatou-se um predomínio do sexo feminino. A literatura destaca que Fortaleza/CE acompanha o crescimento nacional do envelhecimento, visto que as mulheres estão vivendo mais que os homens, entretanto feminização na velhice acarreta as mulheres viverem por maior tempo com adoecimentos crônicos, como hipertensão arterial e diabetes mellitus. Em 2001, 2007 e 2013, houve um aumento significativo de mortes em mulheres com idade superior a 70 anos, devido à maior expectativa de vida destas².

Além disso, autores ${ }^{10}$ registram que as mulheres idosas têm maior probabilidade de incapacidade funcional do que os homens idosos. Uma justificativa apresentada é a relação entre a composição muscular e a força muscular, visto que as mulheres apresentam maior adiposidade muscular e menos força e massa muscular que os homens. Atribui-se também a menor fração de massa muscular entre mulheres à menor prática de atividade física em relação aos homens ${ }^{11}$.

No presente estudo, houve predomínio de idosos com faixa etária inferior (de 60 a 69 anos), destacando-se como possível explicação as dificuldades que o território da UAPS externaliza para locomoção de pessoas com restrição na deambulação, por se tratar de um bairro com muitas moradias fixadas em encostas (morros). Atendimentos domiciliares da eSF e NASF-AB são realizados periodicamente aos idosos que apresentam declínios funcionais da marcha e dificuldade de acesso à unidade.

A característica geográfica do território também influencia no acesso dos moradores aos serviços comerciais e ao transporte público, pois, geralmente, os pontos de ônibus, padarias, supermercados e farmácias ficam distantes dos locais mais altos do morro, ocasionando uma diferenciação do alcance a esses serviços de acordo com a localização da residência do usuário, fato verificado nos achados da pesquisa, em que a minoria reconhece não ter acesso aos mesmos.

O território da população estudada apresenta Índice de Desenvolvimento Humano (IDH) de 0,331. O IDH é um indicador composto pela avaliação da renda, educação e longevidade, com pontuação variando entre 0 e $1^{12}$. Entre os itens pesquisados que apresentam relação com o baixo IDH da região, destaca-se o nível de escolaridade e ocupação/profissão. A maioria dos entrevistados possui entre quatro e sete anos de estudo e exerce ocupações/ profissões com predomínio de trabalhos braçais associadas a baixos salários e serviço informal.

Acerca daseguridadesocial ebenefícios, nota-se que há cobertura de aposentadoria ou pensão para a maioria dos entrevistados. Entre as pessoas que não recebem, quase a metade delas afirmou receber o BPC, que corresponde a renda no valor de um salário-mínimo. Apesar disso, há seis famílias das quais os idosos entrevistados pertencem que recebem auxílio do Programa Bolsa Família, para famílias em situação de pobreza e extrema pobreza.

No Brasil, grande parte da renda dos idosos é oriunda de políticas governamentais e ações assistenciais, não sendo de trabalho renumerado ${ }^{13}$. Registra-se também a relação entre os altos níveis de renda e escolaridade entre idosos com a maior procura por serviços preventivos de saúde, enquanto baixa renda domiciliar e baixa escolaridade relacionam-se com autoavaliações negativas em saúde e piores desempenhos em atividades de vida diária.

Cinco dos entrevistados ainda trabalham, talvez porque não alcançaram o período mínimo de contribuição ou idade ou porque necessitam complementar a renda familiar, fator este que influencia na linha de cuidado do paciente acompanhado na Atenção Primária (AP) de maneiras distintas. A inatividade ou a baixa produtividade laboral, derivadas de transformações na senescência, associadas às incertezas econômicas, geram adoecimentos e transtornos psicossociais que afetam a esfera física. Em contrapartida, a perda da capacidade laborativa, somada à permanência no emprego tardiamente, gera adoecimentos musculoesqueléticos com repercussões crônicas de prognósticos incertos ${ }^{14}$. 
Quanto ao arranjo familiar dos entrevistados, sabe-se que a maioria deles não mora sozinha. A família é fonte primária de suporte e potencializador da saúde física e mental dos idosos, entretanto famílias com maior vulnerabilidade social são mais suscetíveis à confusão de papéis sociais entre os membros, devido às excessivas situações de estresse relacionadas às condições de vida, fragilidades financeiras e violências ${ }^{15}$.

O subsistema conjugal é um dos mais importantes que os indivíduos engajam na vida adulta, com potencial para influenciar positivamente a saúde do casal idoso e garantir bem-estar ${ }^{23}$. A maioria dos entrevistados afirma ser casado, e um percentual maior respondeu positivamente quando perguntado se morava com o(a) cônjuge ou companheiro(a), característica em comum com outras pesquisas que investigaram esse aspecto em público idêntico ${ }^{16,17,18}$.

0 suporte informal se mostrou presente entre os pesquisados, ponto analisado nos itens: "poder contar com a companhia de alguém até a unidade de saúde em caso de urgência" e ter "feito atividade de lazer com amigos ou familiares nos últimos 30 dias". Sabe-se que a participação do indivíduo em atividades de lazer com relações interpessoais com amigos ou familiares favorece uma melhor qualidade de vida ${ }^{19}$.

Dos hábitos de vida dos entrevistados, nota-se predomínio de idosos sedentários ou inativos e minoria que apresenta o hábito de fumar e consumir bebida alcoólica, achado em conformidade com outros estudos que também investigaram 0 estilo de vida de pessoas idosas ${ }^{8,13,16,17,18,20,21}$. Entre os trabalhos que apuraram o consumo anterior de produtos do tabaco e bebidas alcoólicas ${ }^{16,20}$, da mesma forma, observa-se que houve redução dessas práticas entre o público idoso.

No estudo ${ }^{19}$ em que pesquisadores avaliaram a qualidade de vida de 98 idosos vinculados a uma unidade básica de saúde, percebeu-se que exercer atividade física e possuir algum grau de instrução associa-se com melhor percepção da qualidade de vida. Além disso, afirmam que os idosos que participam de atividades com caminhada, fortalecimento muscular, flexibilidade e equilíbrio apresentam melhor qualidade de vida do que os que não participam.
Quanto à avaliação física, nota-se predomínio de sujeitos com excesso de peso entre os entrevistados, dado semelhante ao de pesquisas que investigaram o IMC em idosos em diferentes regiões do país ${ }^{8,16,21}$. Em estudo que avaliou 48 idosas, observou-se correlação negativa entre IMC e força muscular e o IMC foi positivamente correlacionado com maiores níveis de PAS ${ }^{22}$. Os mesmos autores registraram que valores altos de IMC repercutem negativamente na habilidade de subir escadas, caminhar, bem como na independência e bem-estar.

Porém, sabe-se que a probabilidade de ter excesso de peso diminui com o avançar da idade e que perdas superiores a $4,5 \mathrm{~kg}$ ou maiores que $5 \%$ do peso corporal em um ano aumentam de forma acentuada a fragilidade do idoso e podem interferir negativamente em processos de reabilitação ${ }^{21,23}$. Na presente pesquisa, identificou-se apenas uma idosa na situação descrita. Outros autores também encontraram perda de peso não intencional na minoria dos idosos atendidos na $\mathrm{AP}^{24}$.

0 rastreio de condições desencadeadoras de declínio funcional, como elevado IMC ou perda de peso não intencional, é importante para a identificação de sujeitos vulneráveis e propicia intervenções precoces com maior eficácia ${ }^{17}$. Medidas simples, como a verificação da circunferência da panturrilha, podem identificar casos de sarcopenia, conceituada na literatura como perda progressiva e generalizada de massa e função muscular que pode levar a riscos adversos, como incapacidade física, baixa qualidade de vida e morte ${ }^{25}$.

Acrescenta-se que a medida da massa corporal isolada não fornece necessariamente informações sobre disfunções cinético-funcionais ${ }^{26}$. Portanto, relacionaram-se os casos de baixa massa muscular identificados pela medição da circunferência da panturrilha neste estudo com a classificação da pontuação que esses alcançaram no teste de capacidade funcional aplicado: das cinco pessoas que tiveram menos que $31 \mathrm{~cm}$ na medição, três tiveram moderado desempenho e duas, baixo desempenho.

Visualizando a pontuação total do teste de capacidade funcional, a maioria dos avaliados pontuou moderado desempenho. Trabalho que avaliou uma amostra de 195 idosos vivendo em comunidade, de dois sexos, com idade entre 60 e 79 anos, 
utilizando a SPPB, também encontrou predomínio de moderado desempenho $(45,6 \%)$ entre entrevistados $^{9}$, diferentemente do estudo que avaliou, através do mesmo teste, 98 idosos de dois sexos de 60 a 85 anos ou mais, residentes em instituição de longa permanência, e observou predominância de idosos com baixo desempenho (30\%) ${ }^{27}$. Supõe-se, então, que idosos que vivem em comunidades e possuem menor faixa etária, em similaridade com o público da presente pesquisa, tendem a ter meIhores níveis de capacidade física.

Destaca-se a importância de se utilizar a escala SPPB, fragmentada em seus escores, para analisar em quais parâmetros a população apresenta piores resultados e averiguar aspectos físicos específicos $^{11}$. Na literatura, encontraram-se aplicações isoladas de itens diferentes da escala para investigar alterações de equilíbrio e capacidade de manutenção postural em idosos ${ }^{28-29}$.

Analisando os itens separadamente, os idosos pesquisados tiveram pior desempenho (pontuação igual ou menor que três) na terceira etapa, que avaliou a habilidade de sentar e levantar. Ato funcional utilizado em diversas atividades de vida diária e pré-requisito para a mobilidade e independência funcional, quando avaliado, o sentar-levantar permite medir a força em membros inferiores, o equilíbrio corporal, o risco de quedas e a capacidade física para exercícios ${ }^{30}$.

Algumas limitações foram encontradas na realização do presente estudo, como: o número reduzido da amostra; a escolha da amostra, já que se trata de um público com encaminhamento à Fisioterapia provavelmente por queixa ou disfunção identificada inicialmente por profissional de referência; e o delineamento transversal, que impossibilita o estabelecimento de relações de causalidade.

Sugere-se a realização de mais estudos que busquem averiguar a funcionalidade como um conceito ampliado em saúde e a monitorização dos aspectos físicos que comprometem a capacidade física dos sujeitos. Para tanto, ressalta-se a necessidade de distribuição da Caderneta de saúde da pessoa idosa aos usuários do SUS, visto que os entrevistados na pesquisa ainda não possuíam a ferramenta, mesmo depois de dois anos de sua publicação. Acrescenta-se que os mesmos receberam a caderneta logo após a coleta de dados e que esta foi preenchida juntamente com a profissional residente, como também foram dados os devidos encaminhamentos para os casos, levando em consideração as opções de serviços da unidade e o quadro de funcionalidade do usuário.

\section{Conclusão}

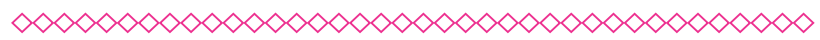

Entre os avaliados, houve predomínio de idosos com moderado desempenho físico, resultado considerado aceitável, já que se trata de idosos que vivem em comunidade. Entretanto, seguidamente, estiveram os entrevistados com baixo desempenho; destes, duas pessoas idosas apresentaram quadro de sarcopenia na avaliação, apresentando um alerta quanto à saúde funcional da população em questão.

Além disso, a maiorparte dosidosos encontra-se em sobrepeso corporal e não pratica atividade física. Quanto aos aspectos sociodemográficos e familiares, ressalta-se a importância do maior aprofundamento, por parte dos fisioterapeutas, no contexto social dos indivíduos atendidos na AP, visto a necessidade de um olhar ampliado e multidimensional sobre a capacidade funcional e a sua interação com o território.

Destaca-se a avaliação da capacidade funcional como recurso para extratificação de idosos em vulnerabilidade física e para o desenvolvimento de ações de intervenção em grupos ou projetos terapêuticos associados ao conhecimento sobre o território em saúde. Sugere-se o mapeamento do nível de capacidade funcional dos demais usuários idosos, a fim de conhecer a situação panorâmica desse público atendido na UAPS em questão.

\section{Referências}

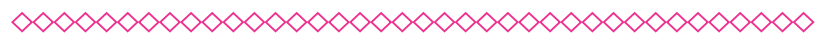

1. Maman B, Barbosa FA, Brugnera A, Silva DB, Scherer RA, Costa JLD, et al. Influência do nível de atividade física na capacidade funcional de mulheres idosas. Revista Destaques Acadêmicos. 2017;9(3):147-54. 
2. Fonsêca Neto MD, Machado CB, Coriolano LS. Fortaleza Cidade Saudável e Fraterna: situação de saúde e perspectiva. Fortaleza: Expressão Gráfica e Editora; 2015.

3. Camarano AA, Kanso S. Envelhecimento da população brasileira: uma contribuição demográfica. In: Freitas EV, Py L. editoras. Tratado de geriatria e gerontologia. 4. ed. Rio de Janeiro: Guanabara Koogan; 2017. p. 181-202.

4. Brasil. Portaria $n^{0} 2.436$ de 21 de setembro de 2017. Aprova a Política Nacional de Atenção Básica, estabelecendo a revisão de diretrizes para a organização da Atenção Básica, no âmbito do Sistema Único de Saúde (SUS). 2017.

5. Ministério da Saúde (BR), Secretaria de Atenção à Saúde, Departamento de Atenção Básica. Práticas em reabilitação na $A B$ : o olhar para a funcionalidade na interação com o território [recurso eletrônico]. Brasília: Ministério da Saúde; 2017.

6. Organização Mundial de Saúde. Direção Geral da Saúde. Classificação Internacional de Funcionalidade, Incapacidade e Saúde. Lisboa; 2004.

7. Ministério da Saúde (BR), Secretaria de Atenção à Saúde, Departamento de Ações Programáticas Estratégicas. Caderneta de saúde da pessoa idosa. 4. ed. Brasília: Ministério da Saúde; 2017.

8. Vagetti GC, Oliveira V, Silva MP, Pacífico $A B$, Costa TRA, Campos W. Associação do índice de massa corporal com a aptidão funcional de idosas participantes de um programa de atividade física. Rev. Bras. Geriatr. Gerontol. 2017;20(2):216-27.

9. Cunha GL, Oliveira GT, Berlese DB, Santos GA. Dano oxidativo ao DNA em idosos com vida independente e suas correlações com parâmetros sociodemográficos, antropométricos e funcionais. Rev. Bras. Geriatr. Gerontol. 2017;20(2):228-35.

10. Biloria BT, Costa AAN, Souza AP, Martins FM, Oliveira AA, Nunes PRP, et al. Maior índice de massa corporal e menor circunferência da cintura estão associados com maior desempenho físico (SPPB) somente em idosas dinapênicas. Acta Fisiatr. 2017;24(1):22-6.
11. Nascimento CR. Estado nutricional antropométrico e a mobilidade funcional de idosos do International Mobility In Aging Study - IMIAS Brasil [Trabalho de Conclusão de Curso]. Natal: Universidade Federal do Rio Grande do Norte; 2019.

12. Secretaria Municipal de Desenvolvimento Econômico (Fortaleza). Desenvolvimento Humano, por bairro, em Fortaleza. 2010. [acesso em 2019 jul 31]. Disponivel em: http:// salasituacional.fortaleza.ce.gov.br:8081/acervo/ documentById?id=22ef6ea5-8cd2-4f96-ad3c8e0fd2c39c98.

13. Borim FSA, Santimaria MR, Moretto MC. Efeitos da pobreza e da desigualdade social sobre a saúde dos idosos. In: Freitas EV, Py L. editoras. Tratado de geriatria e gerontologia. 4. ed. Rio de Janeiro: Guanabara Koogan; 2017. p. 3430-40.

14. Costa GM, Oliveira MLC, Novaes MRCG. Fatores associados à polifarmacoterapia entre idosos assistidos pela estratégia saúde da família. Rev. Bras. Geriatr. Gerontol. 2017;20(4):528-37.

15. Rabelo DF. Aspectos sociais do envelhecimento. In: Freitas EV, Py L. editoras. Tratado de geriatria e gerontologia. 4. ed. Rio de Janeiro: Guanabara Koogan; 2017. p. 3384-98.

16. Corrêa MM, Tomasi E, Thumé E, Oliveira ERA, Facchini LA. Razão cintura-estatura como marcador antropométrico de excesso de peso em idosos brasileiros. Cad. Saúde Pública. 2017;33(5):1-14.

17. Rocha FS, Gardenghi G, Oliveira PC. Perfil de idosos submetidos à avaliação geriátrica ampla em serviço de reabilitação. Rev Bras Promoç Saúde. 2017;30(2):170-8.

18. Pereira LC, Figueiredo MLF, Beleza CMF, Andrade EMLR, Silva MJ, Pereira AFM. Fatores preditores para incapacidade funcional de idosos atendidos na atenção básica. Rev Bras Enferm [Internet]. 2017;70(1):112-8.

19. Oliveira BC, Barbosa NM, Lima MSM, Guerra HS, Neves CM, Avelar JB. Avaliação da qualidade de vida em idosos da comunidade. Rev Bras Promoç Saúde. 2017;30(3):1-10. 
20. Confortin SC, Ono LM, Barbosa AR, D'orsi E. Sarcopenia e sua associação com mudanças nos fatores socioeconômicos, comportamentais e de saúde: Estudo EpiFloripa Idoso. Cad. Saúde Pública. 2018;34(12):1-13.

21. Ferreira CCD, Monteiro GTR, Simões TC. Estado nutricional e fatores associados em idosos: evidências com bases em inquérito telefônico. Rev Bras Promoç Saúde. 2018;31(1):1-10.

22. Nascimento DC, Oliveira OVM, Silva GG, Oliveira ABS, Balsamo S, Tibana RA, et al. Relação entre obesidade, pressão arterial e força muscular de idosas obesas hipertensas. Estud. Interdiscipl. Envelhec. 2017;22(2):9-22.

23. Najas MMAP, Nebuloni CC. Nutrição em Gerontologia. In: Freitas EV, Py L. editoras. Tratado de geriatria e gerontologia. 4. ed. Rio de Janeiro: Guanabara Koogan; 2017. p. 3069-87.

24. Augusti ACV, Falsarella GR, Coimbra AMV. Análise da síndrome da fragilidade em idosos na atenção primária - Estudo transversal. Rev Bras Med Fam Comunidade. 2017;12(39):1-9.

25. Valente M. Sarcopenia. In: Freitas EV, Py L. editoras. Tratado de geriatria e gerontologia. 4. ed. Rio de Janeiro: Guanabara Koogan; 2017. p. 2273-300.
26. Viana LS, Macedo OG, Vilaça KHC, Garcia PA. Concordância de diferentes critérios de sarcopenia em idosas comunitárias. Fisioter Pesqui. 2018;25(2):151-7.

27. Rosa TSM, Santos Filha VAV, Moraes AB. Prevalência e fatores associados ao prejuízo cognitivo em idosos de instituições filantrópicas: um estudo descritivo. Ciência \& Saúde Coletiva. 2018;23(11):3757-65.

28. Bushatsky A, Alves LC, Duarte YAO, Lebrão $M L$. Fatores associados às alterações de equilíbrio em idosos residentes no município de São Paulo em 2006: evidências do Estudo Saúde, Bem-Estar e Envelhecimento (SABE). Rev Bras Epidemiol. 2018;21(Suppl 2):1-14.

29. Silva JCA, Hazime FA, Campelo GO, Silva LN, Ribeiro MDA, Oliveira SB. Capacidade de manutenção postural em diferentes atividades funcionais de idosos hipertensos e não hipertensos. Rev Bras Promoç Saúde. 2017;30(1):22-9.

30. Melo TAM, Duarte ACM, Bezerra TS, França F, Soares NS, Brito D. Teste de sentar-levantar cinco vezes: segurança e confiabilidade em pacientes idosos na alta da unidade de terapia intensiva. Rev Bras Ter Intensiva. 2019;31(1):27. 


\section{Anexos}

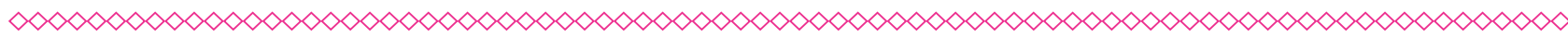

Tabela 1 - Caracterização sociodemográfica e familiar dos entrevistados. Fortaleza, Ceará, Brasil, 2019

\section{Variáveis}

\section{Sexo}

Masculino

Feminino

Idade (anos)

60 a 69 anos

70 a 79 anos

A partir de 80 anos

Raça/cor

Pardo(a)

Branco(a)

Preto(a)

Amarelo(a)

Não declaram raça/cor

Estado civil

Casado(a)

Viúvo(a)

Solteiro(a)

Divorciado(a)

Outro

\section{Escolaridade}

Nenhum ano de estudo

1 a 3 anos

4 a 7 anos

8 anos ou mais

\section{Ocupação/profissão}

Doméstica

Dona de casa

Operária

Artesã

Costureira

Motorista

Pescador

Copeira

Agente administrativo

Agente comunitário de saúde

Cabeleireira

Cozinheira

Cuidadora

\section{N}

$\%$

$$
2
$$

33

5,7

94,3

24

68,6

10

28,6

1

2,8

12

34,3

10

28,6

7

20

1

2,8

14,3

12

34,3

9

25,7

7

20

6

17,2

1

2,8

8

22,8

9

25,7

13

37,1

5

14,2

11

31,4

7

20

4

11,5

3

8,6

5,8

2

2,8

1

2,8

1

2,8

1

2,8

1

2,8

1

2,8

1

2,8

1 


\section{Variáveis}

Trabalha atualmente

Sim

Não

Recebe aposentadoria ou pensão

Sim

Não

Recebe BPC

Sim

Não

Recebe Bolsa Família

Sim

Não

\section{$\mathbf{N}$}

5

30

20

15

8

27

6

29

\section{$\%$}

14,2

85,8

57,1

42,8

22,8

77,2

17,1

82,8

(clique para voltar ao texto) 
Tabela 2 - Caracterização da rede de apoio formal e informal dos entrevistados. Fortaleza, Ceará, Brasil, 2019

\begin{tabular}{lcc} 
Variáveis & N & $\%$ \\
Arranjo familiar & 4 & 11,4 \\
Mora sozinho(a) & 26 & 74,2 \\
Mora com familiar & 13 & 37,1 \\
Mora com cônjuge ou companheiro(a) & & \\
Nos últimos 30 dias se encontrou com amigos ou & & 80 \\
familiares para alguma atividade de fazer & 28 & 20 \\
Sim & 7 & \\
Não & & \\
Conta com alguém para acompanhar à unidade de & 33 & 94,3 \\
saúde ou a uma consulta & 2 & 5,7 \\
Sim & & \\
Não & & 82,8 \\
Fácil acesso aos estabelecimentos comerciais & 29 & 17,2 \\
(farmácia/padaria/supermercado) & 6 & \\
Sim & & 77,2 \\
Não & 27 & 22,8 \\
Fácil acesso a transporte público (ônibus) & 8 & \\
Sim & & \\
Não & & \\
\hline
\end{tabular}


Tabela 3 - Caracterização dos hábitos de vida dos entrevistados. Fortaleza, Ceará, Brasil, 2019

\section{Variáveis \\ Prática de atividade física pelo menos três vezes por semana}

Sim

Não

29

Fuma algum produto de tabaco

Sim

1

Não

Ex-tabagista

Sim

Não

16

47,1

Consome bebida alcóolica

Sim

1

2,8

Não

34

97,2

Anteriormente, fez uso abusivo de bebida alcóolica

Sim 
Tabela 4 - IMC, perda de peso não intencional e circunferência da panturrilha dos entrevistados. Fortaleza, Ceará, Brasil, 2019

\begin{tabular}{lcc} 
Variáveis & N & $\%$ \\
IMC & 2 & 5,7 \\
Baixo peso & 11 & 31,5 \\
IMC adequado & 22 & 62,8 \\
Sobrepeso & & \\
Peso corporal habitual & 10 & 28,5 \\
Não souberam informar o peso corporal habitual & 8 & 32 \\
Peso atual maior que o peso habitual & 1 & 2,8 \\
Perda de peso não intencional maior que 4,5kg & & \\
Circunferência da panturrilha esquerda & 30 & 85,7 \\
Maior que $31 \mathrm{~cm}$ & 5 & 14,3 \\
Menor que $31 \mathrm{~cm}$ & & \\
\hline
\end{tabular}


Tabela 5 - Capacidade funcional entre os entrevistados. Fortaleza, Ceará, Brasil, 2019

\begin{tabular}{lcc} 
Variáveis & N & $\%$ \\
SPPS & 7 & 20,1 \\
Bom desempenho & 18 & 51,4 \\
Moderado desempenho & 8 & 22,8 \\
Baixo desempenho & 2 & 5,7 \\
Incapacidade ou desempenho ruim & & \\
\hline
\end{tabular}

(clique para voltar ao texto) 